\title{
Causas del retardo en el crecimiento fetal intrauterino.
}

Versión tomada de texto en inglés de Conferencia dictada en Universidad Nal. de Colombia.

Dr. Marcos Pupkins Universidad de Duke El concepto de prematurez ligado a bajo peso en el recién nacido e inferior a 2.500 grs, es hoy considerado falso por todos aquellos dedicados al cuidado de él.

Posiblemente ésta ha sido la cáusa para que muchos médicos dejen de reconocer problemas muy especiales ligados a la dismaturidad presente en cualquier periodo del embarazo. La Organización Mundial de la Salud y la Academia Americana de Pediatría, han aprobado el término "niño de peso bajo al nacer" refiriéndose al juzgamiento clínico e histórico de madurez fetal. Desde el punto de vista obstétrico, estamos seguros que continuará la controversia sobre el valor práctico de la fecha probable del parto y el grado de madurez, puesto que el conocimiento de la iniciación del último periodo menstrual de una embarazada dada, depende mucho del grado de inteligencia y de memoria de dicha mujer.

\section{ALGUNOS CONCEPTOS GENERALES}

Al mencionar algunos conceptos generales admitidos como axiomáticos hacemos introducción al tema que nos ocupa.

"Todos los niños pequeños no son prematuros y no deben ser tratados como tales" dice Wilburt Davidson.

"Muchos problemas del recién nacido son imputados al grado de madurez que posee al nacer y están relacionados con la talla" Harry Gordon. Cuando se nos presenta un recién nacido emaciado, cabe la pregunta siguiente: "Porqué éste niño falló dentro de su "habitat" natural uterino?" Ogden C. Burton. Tal pregunta no llega a poseer respuesta en muchos casos. William Crosby afirma: "Si el progreso se logra previniendo partos prematuros y muertos neonatales precoces, tendremos que una muestra del contenido uterino nos indicaría qué tan pronto hemos actuado". Por lo tanto debemos intuir que los parámetros determinantes para juzgar madurez y "status" fetal son extremadamente importantes.
Hace más de una década, se observan niños nacidos con peso bajo y con una inferioridad manifiesta física y mental. Muchos de los cuales no presentan tales alteraciones sino después de meses o aún de años después del nacimiento. Estudios recientes diferenciales entre niños de bajo peso al nacer y auténticos prematuros por talla y edad gestacional mostraron idénticos riesgos y secuelas pos-naturales. El aumento del interés médico por estos problemas y los adelantos logrados para determinar tanto el "status" como la madurez fetales, han facilitado el conocimiento de un retardo en el crecimiento fetal intrauterino.

En éste orden de ideas; un déficit en el peso del nacimiento podrá ser causado: 10 . Por incapacidad del feto para crecer valiéndose de un medio adecuado. 2o. Por parto anticipado, con un peso bajo de acuerdo con la edad gestacional. 3o. Por deprivación, debida a inadecuado suministro de nutrientes maternos o placentarios.

La primera causa es la menos frecuente, en caso de malformaciones o de infecciones crónicas fetales.

La segunda causa de peso al bajo nacimiento, por un parto prematuro, que trae como consecuencia inmadurez funcional orgánica durante el periodo neonatal inmediato. El síndrome de dificultad sería una de las secuelas tardías al igual que las deficiencias de oxigenación con daño cerebral la aparición de alteraciones motoras o mentales. La tercera causa la menos conocida, pero la más común y por lo tanto la más importante es producida por el deficit en el suministro de nutrientes al feto normal durante el embarazo bajo tales circunstancias, se debe comprender al recién nacido desde dos criterios independientes: Peso de nacimiento y edad gestacional. El retardo en el crecimiento existe si el peso del nacimiento está por debajo del límite arbitrario aceptado para la edad gestacional de un niño dado.

Gruenwald ha definido al niño retardado en 
el crecimiento intrauterino, como aquel que pesa dos gradaciones por debajo del peso de su edad gestacional.

Battaglia y Lubchenco los define a los niños de crecimiento retardado como niños de peso bajo que estarían pesando un $10 \%$ por debajo del peso de su edad gestacional.

Tomando las dos definiciones, tenemos que considerar la edad gestacional como aquella determinada por el dato del último periodo menstrual y/o la evaluación neurológica y física del recién nacido.

En cuanto a los efectos de deprivación fetal, éstos han sido divididos en tres estados basándose en su duración.

1) Alteración aguda perinatal presente en el proceso del nacimiento y de duración corta, usualmente de horas.

2) Alteración subaguda, afectando al feto por varios días antes del nacimiento y susceptible de producir baja de peso sin llegar a detectarse un retardo en su crecimiento. El ejemplo de esta modalidad es la distrofia del niño pos-maduro.

3) Deprivación que obra por semanas y el retardo en el crecimiento es ya aparente configurándose una alteración crónica fetal. Las secuelas de la deprivación crónica fetal y el retardo del crecimiento son algo diferentes en el pos-maduro o en el prematuro. En el periodo neo-natal, se presenta hipoglicemia en los neo-natos con retardo del crecimiento posiblemente por deplesión en sus reservas de glicógeno; tal signo tiene gran importancia como causa de daño cerebral. Más tarde en la vida, el retardo del crecimiento puede persistir en algunos niños afectados.

El daño cerebral ha sido reportado, en algunos casos, ocurriendo en niños pequeños por edad gestacional.

La mortalidad perinatal alta es indice útil y definido de dificultades fetales en el análisis estadístico de grupos de población; y el retardo en el crecimiento es la mayor causa de una alta mortalidad perinatal.

En casos de muerte perinatal de 33.40 semanas de gestación, la proporción de niños con peso por debajo del "standar" de desviación para su respectiva edad gestacional, dá un alto índice de muerte perinatal y de mortinatalidad equivalente a diez veces más el de la población general de recién nacidos.
Algunas investigaciones han comparado en estudios longitudinales, el desarrollo del niño a término con el del recién nacido de peso bajo por prematuridad o por retardo en el crecimiento intrauterino.

Tales estudios han demostrado que los niños agrupados por ser pequeños a término, muestran muchos más problemas en su desarrollo que los nacidos a término en quienes se observan disturbios muy similares a los observados en los niños prematuros.

En estudios paralelos a los verificados en el niño normal, tanto el prematuro como el niño con crecimiento retardado, muestran a la edad de ocho años, daño cerebral en un 27.5\%; deficit de peso en 72.5 deficit de talla en un $55 \%$; circunferencia cefálica disminuída $50 \%$ déficit mental en $16 \%$ y disturbios de conducta en el $30 \%$.

En cinco años sucesivos de estudios se ha llaron las siguientes características en ninos de crecimiento retardado intrauterino: deficit neurológico $1 \%$, disfunción mínima cerebral demostrada por hiperactividad, poca coordinación, hipereflexia, dificultad en fijar la atención y dificultad de aprendizaje en el $25 \%$ de los niños en estudio. Fueron halladas anomalías electroencefalográficas $55 \%$; defectos en el lenguaje en $30 \% \mathrm{y} \cdot$ deficientes desempeño escolar en $40 \%$ de aquellos niños que nacieron con retardo en el crecimiento intrauterino.

Las primeras descripciones del síndrome en cuestión, datan de hace setenta años y los nombres adoptados para designarlo fueron: ateliosis fetal, microsom ía fetal, manosomía fetal etc. En los años 50 y 60, en un intento de referirse a factores etiológicos, aparecen en la literatura nuevas denominaciones: enanismo intrauterino, pseudoprematurez, malnutrición fetal, distrofia crónica fetal, disfunción placentaria, insuficiencia placentaria, insuficiencia placentaria y dismaturidad. Las anteriores denominaciones identifica un grupo de niños tenidos por anormales por su discrepancia entre el peso del nacimiento, y la edad gestacional y quienes poseen un gran riesgo de mortalidad perinatal, o de morbilidad, así como de secuelas futuras.

Los niños de crecimiento retardado presentan además disminúción de tejidos blandos, marcado adelgazamiento de la piel, reserva de carbohidratos disminuida, disminución en curva de crecimiento y de la circunferen- 
cia cefálica. Los datos de autopsia de tales niños, muestran que tanto el timo como el hígado son más pequeños que en el niño nacido con peso análogo pero con adecuado crecimiento por edad gestacional, el cerebro es más pesado que el de un pequeño prematuro, pero menos que el de un niño muerto con adecuada edad gestacional.

La incidencia de frecuencia del retardo en el crecimiento intrauterino fluctua entre $2 \%$ a $8 \%$ de nacimientos y depende de su definiciôn.

Entre todos los niños con retardo en el crecimiento intrauterino un $10 \%$ es el producto de embarazos múltiples, $20 \%$ tienen ano malías o infección congénita, $20 \%$ a $30 \%$ poseen madres con infecciones crónicas y 40 a $60 \%$ son productos de embarazos normales.

La etiología del retardo en el crecimiento intrauterino es poco conocida. El concepto de hipoxia fetal prolongada y/o mala nutrición son las bases de varias atractivas hipótesis. La asociación entre retardo en el crecimiento uterino y enfermedad vascular crónica hipertensiva, toxemia del embarazo, enfermedad crónica renal y casos de pacientes con diabetes mellitus es inequivoca.

Es frecuente encontrarlo en pacientes con enfermedades específicas, drogadicción, cardiopatias cianosantes, enfermedad crónica pulmonar, alcoholismo, tirotoxicosis etc. En un porcentaje significativo, de pacientes la alteración puede ser clasificada como idiopática.

Los factores genéticos, pueden atacar a unos pocos niños observados con crecimiento intrauterino retardado. Anomalías cromosómicas tales como trisomias 13-15-18 y 21 dan lugar a anomalías como anencefalia o focomielia pudiendo ser la desafortunada explicación del subnormal crecimiento intrauterino.

Infecciones transplacentarias, procesos de infección crónica intrauterina tales como rubeola, herpes simple, sífilis, toxoplasmosis o enfermedad por inclusiones citomegálicas en algunos casos han sido invocados como agentes etiológicos. El efecto de tales noxas es proporcional a la duración de la infección del feto in útero; tales fetos con el peor pronóstico están ya infectados desde el primer trimestre.

La pequeña talla de los niños con estas infecciones se debe a la disminución del nú- mero de células en muchos órganos, incluyendo cerebro, corazón, hígado, suprarenales y riñones.

Otro factor determinante, es el compromiso de la placenta en el proceso infeccioso; lo cual incluye inflamación de las vellocidades con arteriopatía y esclerosis. En cuanto al mecanismo productor es Ia disminución celular en varios órganos, con inhibición de las mitosis. $\mathrm{Y}$ en no pocos casos aparición de células no viables por alteracio nes cromosómicas. Embriológicamente tales factores serían responsables de alterar el ritmo de crecimiento fetal.

El embarazo prolongado; puede ser también considerado como factor etiológico de estancamiento en el crecimiento in trauterino. Muchos niños teoricamente continuarían ganando peso en la 40a semana de gestación, pero la incidencia de insuficiencia placentaria estaría en aumento al pasar el término; en tal caso no se trataría de verdadero retardo en el crecimiento intrauterino, sino de una pérdida de peso conforme se aleja de la fecha calculada para el parto.

La diabetes ne "natal, raro desorden caracterizado por hipoglicemia, y deshidratación durante la primera semana de vida, ha sido hallada asociada con nacimiento de niños pequeños para su edad gestacional. La causa de tal desorden es desconocida, pero el paso transplacentario al hijo de un anticuerpo materno productor de efecto diabetógeno, ha sido propuesto como causal.

Como factores patológicos determinantes sobre el peso fetal se describen: edad de la madre, talla pequeña materna, paridad materna, sexo del feto, clase social, raza locali zación geográfica y hábito de fumar de la madre

La placenta en niños de crecimiento retardado intrauterino es pequeña y puede presentar infartos múltiples. La correlación èn. tre el retardo de crecimiento intrauterino y enfermedades crónicas vasculares maternas es aparente, siendo facilitado su diagnôstico por el hallazgo pos-mortem de lesiones degenerativas y obliterantes en los vasos espirales del miometrio y desidua, con la consecuente reducción del transporte del sodio intervelloso; contribuyendo todo a reflejar la disminución del flujo sanguíneo utero-placentario. 
En resumen: La etiología del retardo de crecimiento fetal intrauterino es multifactorial, es determinada por múltiples causas no patológicas ya señaladas, como edad, peso, paridad etc. La asociación con enfermedades maternas que alteran el transporte de oxígeno; está demostrado al igual que la mala nutrición. Desde el punto de vista fetal, las infecciones transplacentarias, condiciones genéticas patológicas, además de la deprivación de oxígeno y calorías han sido todas causas que agregadas a la malnutrición fetal e insuficiencia placentaria son causantes del síndrome de retardo en el crecimiento intrauterino.

Con el uso reciente del ultrasonido para valorar el diámetro biparietal y el diámetro transtoráxico del feto afectado, han sido descritos dos grados de retardo de crecimiento: el retardo de crecimiento simétrico comúnmente asociado a reducida ingestión materna de proteínas y el crecimiento retardado asimétrico, aparentemente asociado a insuficiencia vascular utero-placentaria.

\section{DIAGNOSTICO}

La sospecha inicial de un crecimiento intrauterino retardado, depende de la malicia que posea el médico sobre el problema.

Una historia médica y obstétrica completa un examen físico exhaustivo y un meticuloso cuidado pre-natal pueden ser de gran utilidad diagnóstica.

Historia de hipertensión, diabetes mellitus, enfermedad crónica renal, toxemia del embarazo, enfermedad cianótica cardiaca etc., colocan a la paciente en una categoría de alto riesgo con retardo en el crecimiento intrauterino.

La historia obstétrica suministra una información importante en relación con embarazos anteriores, peso del nacimiento, complicaciones prenatales y muertes fetales en el periodo perinatal. Subsecuentemente, 'algunos signos de alarma deben ser tenidos en cuenta por el médico porque le hacen sospechar el síndrome. La presencia de factores de alto riesgo tales como: historia previa de mortinatos, historia de niños pequeños para edad gestacional, niños prematuros anteriores al embarazo en observación, deben ser tenidos en cuenta por el médico como antecedente.

El retardo en el crecimiento fetal intrauterino puede ser sospechado cuando durante el examen físico prenatal continuado, la altura uterina no concuerda con la edad gestacional, o bien cuando durante táles controles se observa crecimiento lento o estacionario uterino; cuando se está en presencia de un oligoamnios; cuando los diámetros biparietales medidos por ultrasonido muestren diámetros menores a los correspondientes a la edad gestacional; y en general cuando el feto dá la impresión de ser más pequeño de lo que se espera por edad gestacional.

Cada vez que se sospeche un crecimiento retardado fetal, debe hacerse el diagnóstico diferencial con un feto inmaduro con adecuado crecimiento para su edad. En un intento para aclarar este punto, debemos de nuevo preguntarnos: Estamos ante un feto de crecimiento retardado, o estamos dando fé a una fecha errada, y el feto se aprecia pequeño porque existe una errónea interpretación de su edad gestacional? Esto debe hacernos pensar, cuan importante es precisar la tal edad gestacional.

Talvez el aspecto más importante de la primera visita, es el estimativo de la edad de embarazo por el tamaño de la matriz y su correlación con la edad gestacional. El examen inicial a fin de establecer dicha edad gestacional debe pues ser verificado por un obstetra-ginecólogo experimentado.

El cálculo de edad por medida de la altura del fondo uterino tiende a ser fallido, sinembargo, la regla del pulgar que dice: "entre las 16 y las 30 semanas de gestación la altura uterina debe estar tantos centímetros por encima de la sinfisis como la edad gestacional en semanas" puede tener algún valor, pero solamente cuando un solo obstetra es quien sigue dicho control.

Durante el curso de control prenatal, una falla en el crecimiento uterino o cualquier discrepancia entre el tamaño de la matriz y la edad gestacional por dato de última regla, debe ser anotado como sospechoso. Pacientes con discrepancia tamaño/fecha en exceso de tres semanas reafirman una sospecha de retardo en el crecimiento intrauterino.

La presencia de polihidramnios u oligoamnios hará sospechar igualmente retardo del crecimiento, especialmente cuando el oligoamnios aparece durante el tercer trimestre. Toda paciente sospechosa debe ser examinada con ultrasonido, pues el crecimiento uterino puede ser seguido tomando las medidas ultrasónicas extrapolares de los diámetros toráxico y biparietal del feto, co- 
rrelacionándolas con peso y edad gestacional. Los resultados de tales medidas han sido considerados practicables y de valor y los fetos que muestran fallas de crecimiento en dos semanas de intervalo deben ser tenidos como de crecimiento retardado.

En el diagnóstico diferencial de la entidad, además de los datos erróneos ya señalados se deben considerar casos en los cuales, niños de talla genéticamente pequeña o fetos normales con diámetros biparietales pequeños al ultrasonido son casos con cabeza del tipo dolicocefálico normal.

En un intento de facilitar el diagnóstico y orientar la conducta a seguir se propone:

1. Historia médica y obstétrica completa

2. Documentación precoz de edad gestacional.

3. Documentación de ayudas que la deter minen

4. Medición clínica de la altura uterina.

5. Cálculo clínico de cant, de líquido amniótico

6. Examen de cervix

7. Estudios ultrasónicos

8. Prueba DHEA

9. Estudios seriados de estrogenos sericos o urinarios

10. Reacción al "Stress" con la ocitocina

11. Determinación de madurez fisiológica fetal

12. Determinación del momento y forma de parto

\section{CONDUCTA}

Toda embarazada con diagnóstico o sospecha de crecimiento uterino retardado, deberá ser trasladada a una clínica prenatal de "alto riesgo" y atendida por experimentados obstetra/ginecólogos, neonatólogos, anestesiólogos y médicos internistas.

En el servicio hospitalario cada paciente deberá ser seguida por un clínico individual con asistencia consultiva inmediata con el resto del grupo médico. La totalidad de exámenes deberán ser hechos no menos de dos veces por semana en las primeras 24 sema- $^{-}$ nas de gestación y luego en forma semanal.

Por lo común, excepto la corrección de enfermedades previas, deficiencias nutrimentales o aconsejar reposo en cama, con el objeto de mejorar la circulación utero placen-: taria; no hay métodos clínicos aceptables para aumentar el crecimiento y mejorar la salud del feto in-utero. La primera dificul- tad en el manejo de la paciente con crecimiento retardado intrauterino consiste en correlacionar una alteración crónica fetal con un gran riesgo de parto e inmadurez que no le favorecerá para sobrevivir.

Para evaluar las condiciones fetales después de la 30a. semana de gestación, semanalmente deberán controlarse estrogenos sericos y urinarios; si la concentración de éstos cae un $50 \%$ en comparación con las determinaciones previas, deberán éstas practicarse diariamente. Tales determinaciones son tomadas con el objeto de diferenciar las bajas espúreas, de las producidas por alteración fetal crónica. Se recomiendan observaciones seriadas con ultrasonido cada 10 a 14 días para establecer el grado de crecimiento del diámetro biparietal. Debe tenerse en cuenta, que estos procedimientos solamente muestran detención en el crecimiento, cuando el feto está severamente comprometido y subsecuentemente un aumento de tales diámetros, no invalida el diagnóstico ya hecho de retardo en el crecimiento intrauterino.

Principiando pronto desde la 32a. semana, la prueba del "stress" con ocitocina debe llevarse a cabo en una semana, con el objeto de tener suficiente documentación de la evolución fetal in utero. Si es necesario, iniciar la vigilancia de la paciente desde las 34 semanas buscando pronta información sobre madurez fetal. Uno de los medios utiles para lograrlo es el estudio llevado a cabo con líquido amniótico tomado por amniocentesis para determinar concentración de creatinina y relación lecitina/esfingomielina.

La presencia de meconio en el líquido amniótico es siempre de pronóstico grave y la terminación del embarazo por el medio más rápido deberá ser la conducta a elegir.

En presencia de indicadores de peligro fetal con o sin evidencia de madurez fetal, el parto deberá llevarse a cabo por la vía más segura. La vía vaginal deberá intentarse en pacientes con posibilidades de parto fácilmente incucible previa evaluación pélvica. La simple amniotomía o en conjunción con einfusión de ocitocina algunas veces estaría, indicada. Existiendo un riesgo significativo de prolapso del cordón. El control por monitor del corazón fetal, del $\mathrm{Ph}$ craneano fetal se emplean durante el proceso del parto. En especial la monitoria debe ser rutina en estos casos. Dejando el control bioquímico 
para casos especiales. En caso de sufrimiento fetal agudo demostrable durante el parto, la cesárea es imperativa y es el método más seguro inclusive en el caso de dificultad de inducción $y$ premura en la terminación del parto.

Teniendo en cuenta la modalidad de parto, el pediatra que ha intervenido en la toma de decisiones deberá estar presente en la sala. Estando plenamente demostrada la ocurrencia de acidosis neonatal, hipoglicemia y hemorragia pulmonar en el neonato de crecimiento retardado se justifica prepararse con anticipación para conjurar tales problemas.

\section{SUMMARY}

In summary, the etiology of intrauterine growth retardation is multifactorial, there is a multiplicity of non-pathologic determinants as maternal age, weight, parity, sex, race, etc. The association to maternal illness that impair oxigen transport as well as malnutrition are well documented. From the fetal point of view transplacental infections, pathological genetic conditions, in adition to intrauterin fetal deprivation of oxigen and calories have been described in association to fetal malnutrition and placental insuficiency as part of the syndrome of intrauterine growth retardation. 